\title{
磺酰氯参与的基于远端炔基迁移的非活化烯烃炔基化反应
}

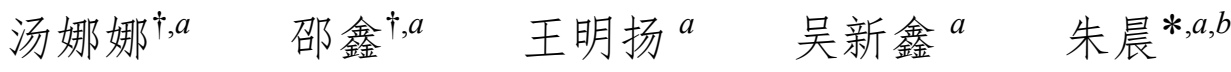 \\ ( (苏州大学 材料与化学化工学部 苏州 215123) \\ $\left({ }^{b}\right.$ 中国科学院上海有机化学研究所 天然产物有机合成重点实验室 上海 200032)
}

\begin{abstract}
摘要 利用自由基参与的远端炔基迁移策略实现了非活化烯烃的炔基化反应. 以磺酰氯为试剂, 在可见光促进下形成 三氟甲基自由基或芳基(烷基)磺酰基自由基，随后加成到非活化烯烃上，生成烷基自由基中间体，继而诱导远端炔烃的 分子内迁移，得到三氟甲基炔基化或磺酰基炔基化产物. 该方法具有条件温和、底物易得以及官能团兼容性广等优点. 关键词 炔基化; 三氟甲基化; 磺酰化; 烯烃双官能团化; 官能团迁移
\end{abstract}

\section{Sulfonyl Chlorides Mediated Alkynylation of Non-activated Alkenes via Distal Alkynyl Group Migration}

\author{
Tang, Nana ${ }^{\dagger, a} \quad$ Shao, Xin ${ }^{\dagger, a} \quad$ Wang, Mingyang $^{a} \quad \mathrm{Wu}$, Xinxin $^{a} \quad$ Zhu, Chen ${ }^{*, a, b}$ \\ $\left({ }^{a}\right.$ College of Chemistry, Chemical Engineering and Materials Science, Soochow University, Suzhou, Jiangsu 215123) \\ $\left({ }^{b}\right.$ Key Laboratory of Synthetic Chemistry of Natural Substances, Shanghai Institute of Organic Chemistry, \\ Chinese Academy of Sciences, Shanghai 200032)
}

\begin{abstract}
Radical-mediated difunctionalization of alkenes through the remote functional group migration (FGM) process paves an ingenious avenue for simultaneous cleavage and reconstruction of $\mathrm{C}-\mathrm{C}$ bonds. Recently, our group has systematically disclosed the strategy of remote FGM for radical-mediated difunctionalization of unactivated alkenes. A portfolio of functional groups including cyano, heteroaryl, alkynyl, oximino, and carbonyl showcase the migratory aptitude, leading to new $\mathrm{C}-\mathrm{C}$ bonds under radical conditions. Meanwhile, a series of other chemical bonds such as $\mathrm{C}-\mathrm{C}, \mathrm{C}-\mathrm{N}, \mathrm{C}-\mathrm{P}, \mathrm{C}-\mathrm{Si}$, and $\mathrm{C}-\mathrm{S}$ are readily constructed in the reaction. Considering the synthetic utility and flexible transformation of alkynes, the radical alkynylation of unactivated alkenes via remote alkynyl migration we firstly reported provides an efficient approach for the incorporation of alkynes and has received considerable attention. On the other hand, fluorine- and sulfonyl-containing molecules play vital roles in organic and medicinal chemistry owing to their important chemical and physical characters. Therefore, the concomitant introduction of an alkynyl and a trifluoromethyl/sulfonyl group into one molecule is of great synthetic value. Herein we report a useful method for carbo-trifluoroalkylation/sulfonylation of unactivated olefins. The addition of extrinsic radical to alkene triggers the intramolecular FGM via a five-membered transition state along with a cascade of bond fission and formation. The typical procedure is as follows: a mixture of propargyl alcohol 1, sulfonyl chloride, $f a c-\operatorname{Ir}(\mathrm{ppy})_{3}$, and base is loaded in a flame-dried reaction vial which is subjected to evacuation/flushing with nitrogen three times. Solvent is added to the mixture via syringe and the mixture is then stirred at $25{ }^{\circ} \mathrm{C}$ until the starting material is consumed monitored by TLC. The mixture is concentrated, and purified by flash column chromatography on silica gel (eluent: petroleum ether/ethyl acetate) to give the product.
\end{abstract}

Keywords alkynylation; trifluoromethylation; carbosulfonylation; alkene difunctionalization; functional group migration

\section{1 引言}

在有机合成中, $\mathrm{C}-\mathrm{C}$ 键的断裂和构建是至关重要 的 ${ }^{[1]}$. 自由基促进的分子内远端官能团迁移(FGM)过程 实现了非活化烯烃的双官能团化, 同时为构建 $\mathrm{C}-\mathrm{C}$ 键 提供了一条新的途径 ${ }^{[2,3]}$. FGM 的基本历程是, 首先自由 基加成到末端烯烃上形成烷基自由基中间体, 再通过五 元环或六元环过渡态引发分子内官能团 1,4 或 1,5-迁移. 在此过程中伴随着 $\mathrm{C}-\mathrm{C}$ 键的断裂和新的 $\mathrm{C}-\mathrm{C}$ 键的形
成. 最近, 我们小组系统地研究了自由基促进的基于远 端官能团迁移策略实现的非活化烯烃的双官能化反应, 其中包括氧基 ${ }^{[2 \mathrm{c}, 2 \mathrm{e} \sim 2 \mathrm{f}, 2 \mathrm{~m}]}$ 、杂芳基 ${ }^{[2 \mathrm{~d}, 2 \mathrm{~g} \sim 21]}$ 、炔基 ${ }^{[2 \mathrm{~h}, 4]}$ 、亚 胺 ${ }^{[2 \mathrm{~h}, 2 \mathrm{j}, 2 \mathrm{2}]}$ 和酫 ${ }^{[2 \mathrm{~h}]}$ 在内的一系列官能团均表现出较好的迁 移能力(图 1a). 采用该策略在构建 $\mathrm{C}-\mathrm{C}$ 键的同时还能 构建另外一根化学键, 例如 $\mathrm{C}-\mathrm{C} 、 \mathrm{C}-\mathrm{N} 、 \mathrm{C}-\mathrm{P} 、 \mathrm{C}-$ $\mathrm{Si}$ 或 $\mathrm{C}-\mathrm{S}$ 键等.

炔基化合物因具有潜在的可修饰性从而使其在化

\footnotetext{
*E-mail: chzhu@suda.edu.cn; Tel.: 0512-65883179

$\dagger$ These authors contributed equally.

Received May 1, 2019; published June 4, 2019

Supporting information for this article is available free of charge via the Internet at http://sioc-journal.cn.

Project supported by the National Natural Science Foundation of China (No. 21722205).

项目受国家自然科学基金(No. 21722205)资助.
} 
学合成中具有广泛的应用. 我们小组首次报道了通过远 端炔基迁移实现的非活化烯烃的三氟甲基炔基化反应， 为炔基化合物合成提供了一种有效的途径，并受到了广 泛的关注 ${ }^{[4]}$. 随后, 通过此策略实现非活化烯烃的全氟 烷基炔基化、二氟烷基炔基化和烷基炔基化等反应被相 继报道 ${ }^{[5]}$ (图 1b). 由于磺酰氯具有廉价易得等优势, 同 时砜类化合物具有显著的生物活性 ${ }^{[6]}$, 在有机化学和药 物化学中发挥着重要的作用, 所以磺酰基和炔基的同时 引入具有重要的合成价值. 因此我们选择以磺酰氯作为 自由基源，系统地考察了芳基磺酰氯、烷基磺酰氯以及 三氟甲基磺酰氯在非活化烯烃的炔基化反应中反应效 果, 以此成功构建了 $\beta$ 位砜基以及含氟烷基取代的链状 炔烃(图 1c).

(a) radical difunctionalization of alkenes via remote FGM<smiles>[R]CC(F)CC([R])CC(=O)O</smiles>

FG = cyano, heteroaryl, alkynyl, oximino, carbonyl ...

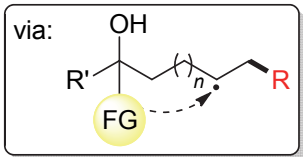

(b) previous work

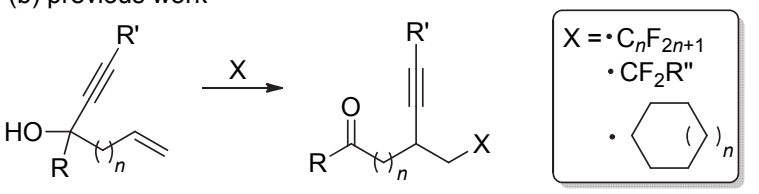

(c) this work<smiles>[R]C#CC([R])(O)CC=C</smiles><smiles>[R7][R7]#[R6]CC(C#C[R])CNC([R])=O</smiles>

图 1 通过远端官能团迁移策略实现非活化烯烃的双官能团化 Figure 1 Radical-mediated difunctionalization of unactivated alkenes via remote FGM

\section{2 结果与讨论}

\section{1 芳基磺酰氯及烷基磺酰氯参与的炔基化反应}

\section{1 .1 条件耖选}

以炔丙醇 (1a)作为模板底物, 4-甲基苯磺酰氯 $(\mathrm{TsCl})$ 作为自由基源, 对该反应进行了初步篎选, 实验结果如 表 1 所示. 在以 1.0 equiv. 的碳酸钠作为碱, 乙腈作为反 应溶剂的条件下, 我们对光催化剂进行了篎选(Entries $1 \sim 4)$. 当以 $3 \mathrm{~mol} \% f a c-\operatorname{Ir}(p p y)_{3}$ 作为光敏剂时, 可以得 到较好的产率(Entry 4). 另外, 我们也对碱的用量进行 了笁选(Entries 4 6), 实验结果表明, 1.0 equiv. 时效果 最佳, 增加或减少碱的用量都不利于反应. 对反应溶剂
笁选后，我们发现：在以丙酮作为溶剂时，产率稍有降 低(Entry 7); 二氯甲烷作为反应溶剂时，只能以中等产 率得到目标产物(Entry 8); 当以二甲亚砜和 $N, N$-二甲基 甲酰胺作反应溶剂时，TLC 检测只有少量产物生成，同 时反应体系中原料 1a 大量剩余(Entries 9, 10). 最终我们 确定表 1 中的 Entry 4 为最优条件.

表 1 反应条件的优化 ${ }^{a}$

Table 1 Reaction condition optimization ${ }^{a}$

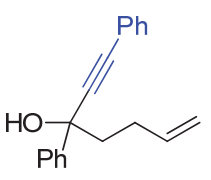

$1 \mathrm{a}$

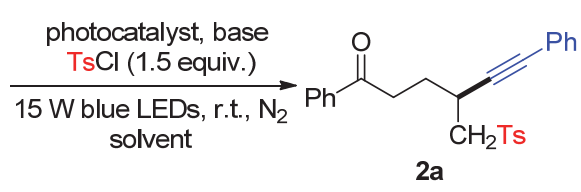

$2 a$

\begin{tabular}{cllll}
\hline Entry & \multicolumn{1}{c}{ Photocatalyst $(3 \mathrm{~mol} \%)$} & $\mathrm{Base}(x$ equiv. $)$ & Solvent & Yield/\% \\
\hline 1 & $\mathrm{Ir}\left\{\left[\mathrm{dF}\left(\mathrm{CF}_{3}\right) \mathrm{ppy}\right]_{2}-(\mathrm{dtbbpy})\right\} \mathrm{PF}_{6}$ & $\mathrm{Na}_{2} \mathrm{CO}_{3}(1.0)$ & $\mathrm{CH}_{3} \mathrm{CN}$ & $<5$ \\
2 & $\operatorname{Ir}(\text { ppy })_{2}(\mathrm{dtbbpy}) \mathrm{PF}_{6}$ & $\mathrm{Na}_{2} \mathrm{CO}_{3}(1.0)$ & $\mathrm{CH}_{3} \mathrm{CN}$ & $<5$ \\
3 & $\mathrm{Ru}(\mathrm{bpy})_{3} \mathrm{Cl}_{2} \cdot 6 \mathrm{H}_{2} \mathrm{O}$ & $\mathrm{Na}_{2} \mathrm{CO}_{3}(1.0)$ & $\mathrm{CH}_{3} \mathrm{CN}$ & $<5$ \\
4 & $f a c-\operatorname{Ir}(\mathrm{ppy})_{3}$ & $\mathrm{Na}_{2} \mathrm{CO}_{3}(1.0)$ & $\mathrm{CH}_{3} \mathrm{CN}$ & 75 \\
5 & $f a c-\operatorname{Ir}(\mathrm{ppy})_{3}$ & $\mathrm{Na}_{2} \mathrm{CO}_{3}(0.8)$ & $\mathrm{CH}_{3} \mathrm{CN}$ & 57 \\
6 & $f a c-\operatorname{Ir}(\mathrm{ppy})_{3}$ & $\mathrm{Na}_{2} \mathrm{CO}_{3}(1.2)$ & $\mathrm{CH}_{3} \mathrm{CN}$ & 68 \\
7 & $f a c-\operatorname{Ir}(\mathrm{ppy})_{3}$ & $\mathrm{Na}_{2} \mathrm{CO}_{3}(1.0)$ & $\mathrm{acetone}$ & 68 \\
8 & $f a c-\operatorname{Ir}(\mathrm{ppy})_{3}$ & $\mathrm{Na}_{2} \mathrm{CO}_{3}(1.0)$ & $\mathrm{DCM}$ & 58 \\
9 & $f a c-\operatorname{Ir}(\mathrm{ppy})_{3}$ & $\mathrm{Na}_{2} \mathrm{CO}_{3}(1.0)$ & $\mathrm{DMSO}$ & $<10$ \\
10 & $f a c-\operatorname{Ir}(\mathrm{ppy})_{3}$ & $\mathrm{Na}_{2} \mathrm{CO}_{3}(1.0)$ & $\mathrm{DMF}$ & $<10$ \\
\hline
\end{tabular}

${ }^{a}$ Reaction conditions: 1a $(0.2 \mathrm{mmol})$, solvent $(2 \mathrm{~mL})$, at r.t. and under $\mathrm{N}_{2}$ atmosphere. Yields of isolated products are given.

\section{1 .2 反应底物拓展}

在确定最优反应条件后, 我们对底物适用性进行考 察(图 2). 在苯环邻位、间位、对位有甲基取代时, 反应 均能以较好收率得到炔基迁移产物 $(2 \mathrm{~b}, 2 \mathrm{e}$ 和 $2 \mathrm{~g})$. 另外, 苯基的 3,5 位同时有甲基取代时，反应也能取得良好收 率(2f). 当苯基上有吸电子基如氯、溴取代时，反应也能 获得中等收率(2c，2d 和 2h). 另外，当用正戊基代替芳 基时，该反应也能以稳定的收率得到目标产物(2i). 这 也说明，无论是芳基还是烷基取代的三级炔丙醇都适用 于该反应体系. 然后我们考察了芳基炔上连有不同取代 基时对反应的影响. 无论是给电子基团还是吸电子基团 取代的芳基, 均能转化为相应的产物 $(\mathbf{2} \mathbf{j} \sim \mathbf{2 m})$, 但是连 有吸电子基团时，产率略有降低. 接下来，我们对连接 不同取代基的芳基磺酰氯进行考察. 结果表明，取代基 的电性以及取代位置对反应的发生几乎没有影响，且均 能以中等到良好的收率得到目标产物 $(\mathbf{2 n} \sim \mathbf{2 q})$. 最后, 我们发现，当用烷基磺酰氯代替芳基磺酰氯时，反应依 然能够进行 (2r 和 $2 s$ ). 但是当以异丙基磺酰氯作为底物 时, 反应收率有所降低. 其原因可能是, 异丙基的推电 子效应对反应效果造成了负面影响. 除 1,4-炔基迁移外, 我们也能以较低产率实现 1,5-炔基迁移反应(2t). 但是 其它类型的 $1, n$-炔基迁移反应 $(n=2,3,6$ 等)在此反应条 件下未能取得成功. 


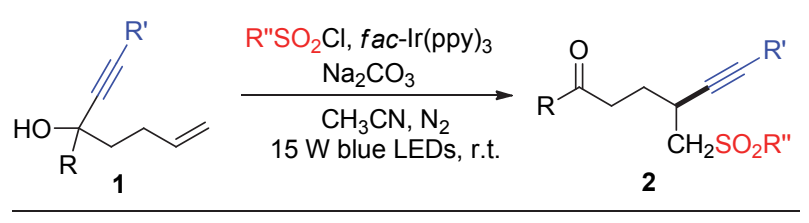<smiles>[R]c1ccc(C(=O)CCC(C#CPc2ccccc2)C[15F])cc1</smiles>

$\mathrm{R}=\mathrm{H}, \quad \mathbf{2 a}, 75 \%, 6 \mathrm{~h}$ $\mathrm{R}=\mathrm{Me}, \mathbf{2 b}, 70 \%, 6 \mathrm{~h}$

$\mathrm{R}=\mathrm{Cl}, \mathbf{2 c}, 41 \%, 6 \mathrm{~h}$

$\mathrm{R}=\mathrm{Br}, \mathbf{2 d}, 31 \%, 6 \mathrm{~h}$

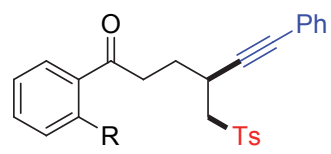

$\mathrm{R}=\mathrm{Me}, \mathbf{2 g}, 60 \%, 6 \mathrm{~h}$

$\mathrm{R}=\mathrm{Cl}, \quad 2 \mathrm{~h}, 57 \%, 6 \mathrm{~h}$

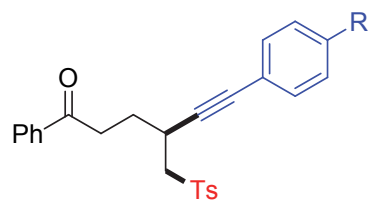

$\mathrm{R}=\mathrm{Me}, \mathbf{2 j}, 63 \%, 4 \mathrm{~h}$

$\mathrm{R}=\mathrm{CF}_{3}, \mathbf{2 k}, 45 \%, 7 \mathrm{~h}$<smiles>O=C(CCC(C#Cc1ccccc1F)C[15F])c1ccccc1</smiles>

$2 \mathrm{~m}, 60 \%, 6 \mathrm{~h}$<smiles>Cc1cccc(S(=O)(=O)CC(C#Cc2ccccc2)CCC(=O)c2ccccc2)c1</smiles>

2p, $65 \%, 5$ h<smiles>[R]S(=O)(=O)CC(C#Cc1ccccc1)CCC(=O)c1ccccc1</smiles>

$\mathrm{R}=\mathrm{Me}, \mathbf{2 r}, \mathbf{7 3} \%, 5 \mathrm{~h}$

$\mathrm{R}={ }^{i} \operatorname{Pr}, \mathbf{2 s}, 35 \%, 6 \mathrm{~h}$

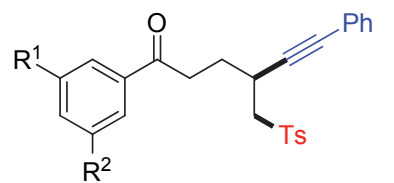

$\mathrm{R}^{1}=\mathrm{H}, \mathrm{R}^{2}=\mathrm{Me}, \mathbf{2 e}, 65 \%, 6 \mathrm{~h}$ $R^{1}=M e, R^{2}=M e, 2 f, 70 \%, 4 h$<smiles>CCCCC(=O)CCC(C#Cc1ccccc1)C[18F]</smiles>

2i, $40 \%, 9$ h<smiles>Cc1cccc(C#CC(C[18F])CCC(=O)c2ccccc2)c1</smiles>

2I, $64 \%, 4 \mathrm{~h}$<smiles>[R]c1ccc(S(=O)(=O)CC(C#Cc2ccccc2)CCC(=O)c2ccccc2)cc1</smiles>

$\mathrm{R}=\mathrm{Cl}, \quad 2 \mathrm{n}, 54 \%, 7 \mathrm{~h}$

$\mathrm{R}=\mathrm{CF}_{3}, \mathbf{2 0}, 35 \%, 5 \mathrm{~h}$<smiles>Cc1ccccc1S(=O)(=O)CC(C#Cc1ccccc1)CCC(=O)c1ccccc1</smiles>

2q, $52 \%, 7 \mathrm{~h}$

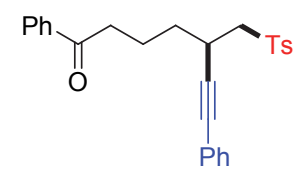

2t, $27 \%, 6 \mathrm{~h}$

图 2 底物拓展

Figure 2 Substrate scope

Reaction Conditions: propargyl alcohol $1(0.2 \mathrm{mmol}), \mathrm{Na}_{2} \mathrm{CO}_{3}(0.2 \mathrm{mmol}, 1.0$ equiv.), $f a c-\operatorname{Ir}(\mathrm{ppy})_{3}(0.006 \mathrm{mmol}, 3 \mathrm{~mol} \%)$, and sulfonyl chloride $(0.3 \mathrm{mmol}$, 1.5 equiv.), irradiated with $15 \mathrm{~W}$ blue LEDs at room temperature under $\mathrm{N}_{2}$ atmosphere. Yields of isolated products are given.

\section{2 三氟甲磺酰氯参与的炔基化反应}

\subsection{1 条件篮选}

首先, 以 $\mathrm{CF}_{3} \mathrm{SO}_{2} \mathrm{Cl}$ 和炔丙醇 $\mathbf{1 a}$ 作为模板底物, $f a c-\operatorname{Ir}(\mathrm{ppy})_{3}$ 作为光催化剂, $\mathrm{K}_{2} \mathrm{HPO}_{4}$ 作为添加剂, 在室温

和 $15 \mathrm{~W}$ 的蓝色 LED 光照射下优化反应条件(表 2). 有机 溶剂篎选(Entries 1 5)的结果表明, 当以丙酮作为反应 溶剂时，以相对较好的收率得到目标产物 3a (Entry 2). 由于碱可以中和反应产生的 $\mathrm{HCl}$ 从而抑制消除反应等 副反应的进行，随后我们对添加碱的种类和用量进行仔 细地篎选 (Entries $6 \sim 12$ ). 相较于其他无机碱 (如 $\mathrm{Na}_{2} \mathrm{HPO}_{4} 、 \mathrm{Na}_{2} \mathrm{CO}_{3} 、 \mathrm{~K}_{2} \mathrm{CO}_{3}$ 和 $\mathrm{NaHCO}_{3}$ ) 或有机碱(如 $\mathrm{DABCO}$ ), 添加 0.8 equiv.的 $\mathrm{K}_{2} \mathrm{HPO}_{4}$ 时效果最好. 另外, 将光催化剂的用量提高到 $4 \mathrm{~mol} \%$ 时, $3 \mathrm{a}$ 的产率略有提 高(Entry 13). 尽管增加 $\mathrm{CF}_{3} \mathrm{SO}_{2} \mathrm{Cl}$ 的用量且将其一次性 投入反应体系中，反应产率并没有得到明显的提升 (Entry 14), 但是通过分批加入 2.2 equiv. 的 $\mathrm{CF}_{3} \mathrm{SO}_{2} \mathrm{Cl}$ 则 可以显著提高反应产率(Entry 15). 此外, 当 $f a c-\operatorname{Ir}(\mathrm{ppy})_{3}$ 被其他光催化剂(如 $\mathrm{Ru}(\mathrm{bpy})_{3} \mathrm{Cl}_{2} \cdot 6 \mathrm{H}_{2} \mathrm{O} 、 \operatorname{Ir}(\mathrm{ppy})_{2}$ (dtbbpy)$\mathrm{PF}_{6}$ 等)所替代时, 则未检测到目标产物 $3 \mathrm{a}$ 的生成 (Entries 16,17). 最后, 控制实验结果表明, 在没有光催 化剂或可见光照射时, 反应无法进行(Entries 18, 19).

\subsection{2 反应底物拓展}

在确定最优条件后, 对底物适用性进行考察(图 3). 在该反应体系中，各种供电子官能团(如甲基和叔丁基 等)和吸电子官能团(如卤素、三氟甲基和三氟甲氧基等) 均表现出较好的兼容性. 富电子基团取代的芳基炔丙醇 通常比缺电子基取代的芳基炔丙醇以相对较高的收率 转化为目标产物. 其中, 溴取代的目标产物(3f)还可以 通过偶联反应用于后期修饰，以转化得到其他具有合成 价值的化合物. 此外，烷基(如正戊基、环己基和芐基) 取代的炔丙醇也能顺利地转化为多官能团化的二烷基 酮(3m 3o). 最后, 我们对迁移官能团芳基乙炔上的取 代基进行了考察. 结果表明, 缺电子和富电子官能团取 代的芳基乙炔均能顺利实现迁移且得到目标产物(3p 3v), 而且缺电子苯乙炔的迁移效率稍优于富电子苯乙 炔. 造成该结果的原因可能是，原位产生的亲核性烷基 自由基更倾向于进攻电子云密度较低的不饱和碳碳三 键.

\section{3 机理与讨论}

根据实验结果以及前期的相关研究工作报道, 我们 提出了如下可能的机理路径(图 4). 首先激发态的 Ir III 光 敏剂被磺酰氯氧化性淬灭, 生成 $\operatorname{Ir}^{\mathrm{IV}}$ 络合物及砜自由基. 但是, 三氟甲基砜自由基会进一步脱除 $\mathrm{SO}_{2}$ 得到三氟甲 基自由基. 随后, 砜自由基或三氟甲基自由基加成到炔 丙醇底物的末端烯烃上得到烷基自由基中间体 I. 后者 被分子内的炔烃所捕获, 经过五元环过渡态 II 以及随后 的环状碳碳键断裂, 从而实现炔基的远端迁移, 得到亚 稳态自由基中间体 III. 随后，中间体 III 被 $\mathrm{Ir}^{\mathrm{IV}}$ 络合物 氧化生成碳正离子中间体 IV, 再经过脱质子, 得到目标 产物 2 或 3. 

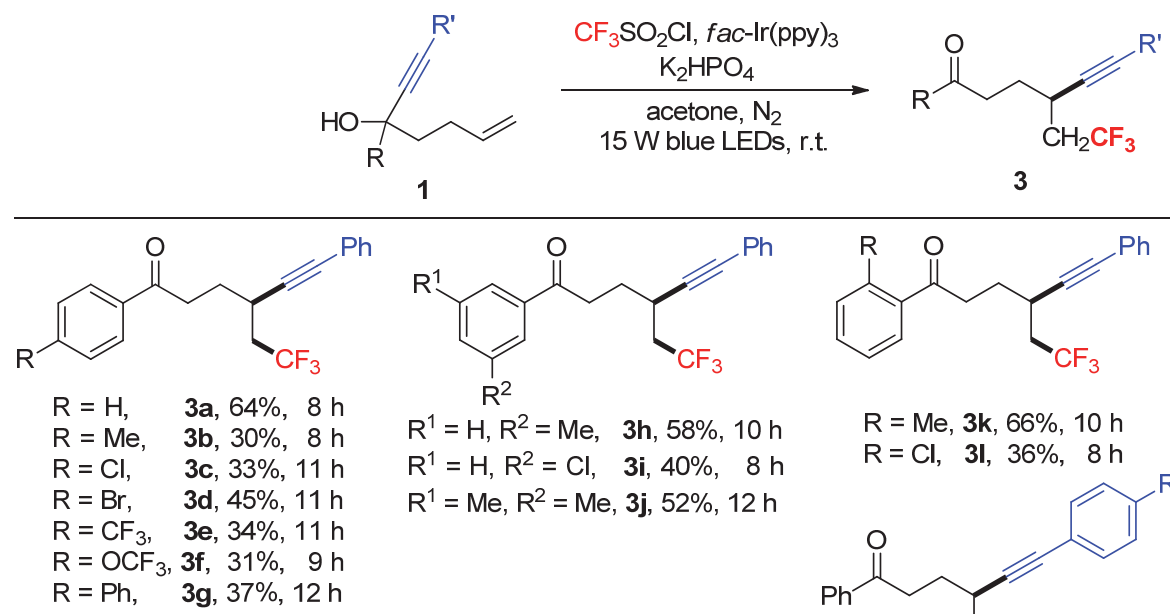

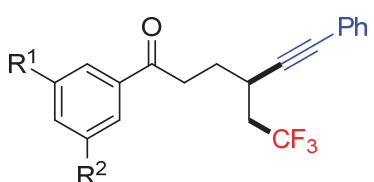

$\mathrm{R}^{1}=\mathrm{H}, \mathrm{R}^{2}=\mathrm{Me}, \quad 3 \mathrm{~h}, 58 \%, 10 \mathrm{~h}$

$\mathrm{R}^{1}=\mathrm{H}, \mathrm{R}^{2}=\mathrm{Cl}, \quad 3 \mathrm{i}, 40 \%, \quad 8 \mathrm{~h}$

$R^{1}=M e, R^{2}=M e, 3 j, 52 \%, 12 h$<smiles>O=C(CCC(C#Cc1ccccc1)CC(F)(F)F)c1ccccc1</smiles>

3o, $27 \%, 10 \mathrm{~h}$<smiles>[R]c1ccccc1C(=O)CCC(C#Cc1ccccc1)CC(F)(F)F</smiles>

$\mathrm{R}=\mathrm{Me}, 3 \mathbf{k}, 66 \%, 10 \mathrm{~h}$ $\mathrm{R}=\mathrm{Cl}, 3 \mathrm{I}, 36 \%, 8 \mathrm{~h}$<smiles>[R]c1ccc(C#CC(CCCCC)CC(F)(F)F)cc1</smiles>

$\mathrm{R}=\mathrm{F}, \quad 3 \mathrm{p}, 35 \%, 11 \mathrm{~h}$

$\mathrm{R}=\mathrm{Cl}, \quad 3 \mathrm{q}, 54 \%, 10 \mathrm{~h}$

$\mathrm{R}=\mathrm{CF}_{3}, \quad 3 \mathbf{r}, 50 \%, 11 \mathrm{~h}$

$\mathrm{R}={ }^{t} \mathrm{Bu}, \quad 3 \mathrm{~s}, 40 \%, 11 \mathrm{~h}$<smiles>CCCCC(=O)CCC(C#Cc1ccccc1)CC(F)(F)F</smiles>

$3 \mathrm{~m}, 57 \%, 9 \mathrm{~h}$<smiles>O=C(CCC(C#Cc1ccccc1F)CC(F)(F)F)c1ccccc1</smiles>

$3 t, 51 \%, 11 \mathrm{~h}$

3n, $34 \%, 9$ h<smiles>Cc1cccc(C#CC(CCC(=O)c2ccccc2)CC(F)(F)F)c1</smiles>

$3 u, 35 \%, 12 h$<smiles>CCCCC(=O)c1ccc(C#CC(CCC(=O)c2ccccc2)CC(F)(F)F)cc1</smiles>

$3 v, 53 \%, 11 \mathrm{~h}$

图 3 底物拓展

Figure 3 Substrate scope

Reaction Conditions: propargyl alcohol $1(0.2 \mathrm{mmol}), \mathrm{K}_{2} \mathrm{HPO}_{4}(0.16 \mathrm{mmol}, 0.8$ equiv. $)$, $f a c-\mathrm{Ir}(\mathrm{ppy})_{3}(0.008 \mathrm{mmol}, 4 \mathrm{~mol} \%)$, and $\mathrm{CF}_{3} \mathrm{SO}_{2} \mathrm{Cl}(0.44 \mathrm{mmol}, 2.2 \mathrm{equiv}$. in two portions), irradiated with $15 \mathrm{~W}$ blue LEDs at r.t. under $\mathrm{N}_{2}$ atmosphere. Yields of isolated products.<smiles>[R]C#CC(C[Y])CC([R])=O</smiles><smiles>[R]C#CC(C[X])CC([R])O</smiles>

IV

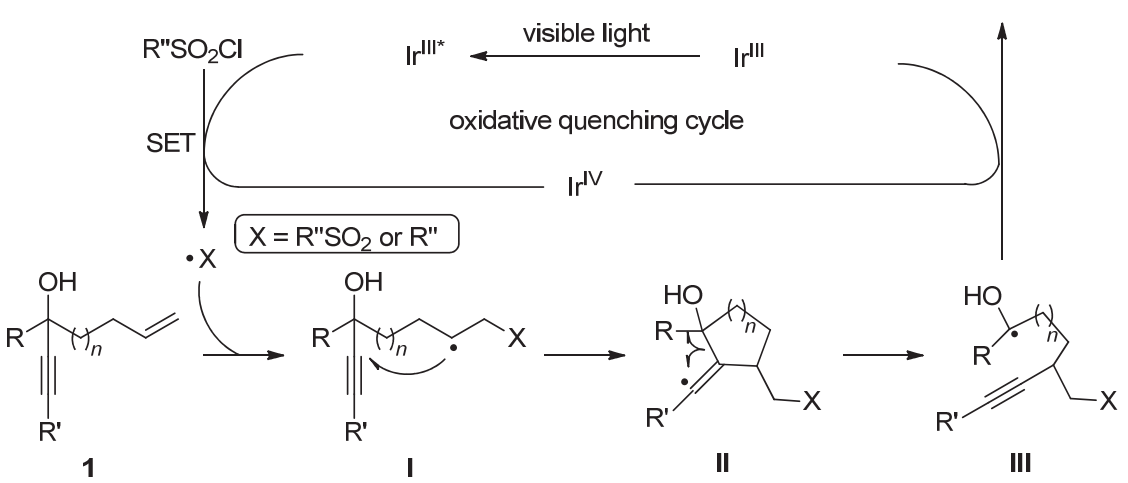

图 4 机理推测

Figure 4 Proposed mechanism

\section{4 结论}

综上所述, 我们以廉价易得的磺酰氯试剂作为自由 基源，在可见光促进下，利用远端炔基的分子内迁移策 略实现了非活化烯烃的炔基化和三氟甲基/磺酰化反应.
该反应条件温和，且具有良好的官能团耐受性. 通过该 策略得到大量的多官能团化的 $\gamma$-炔酮, 这类产物还可用 于后续的分子修饰以合成其它具有更高应用价值的化 合物. 
表 2 反应条件的优化 ${ }^{a}$

Table 2 Reaction condition optimization ${ }^{a}$

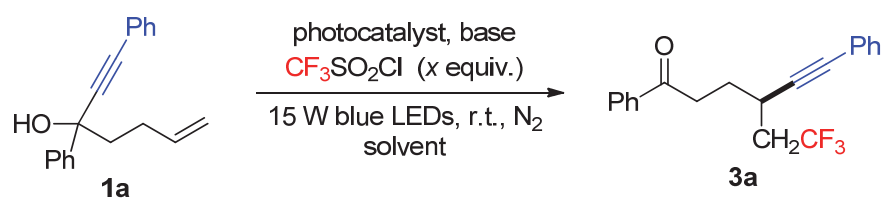

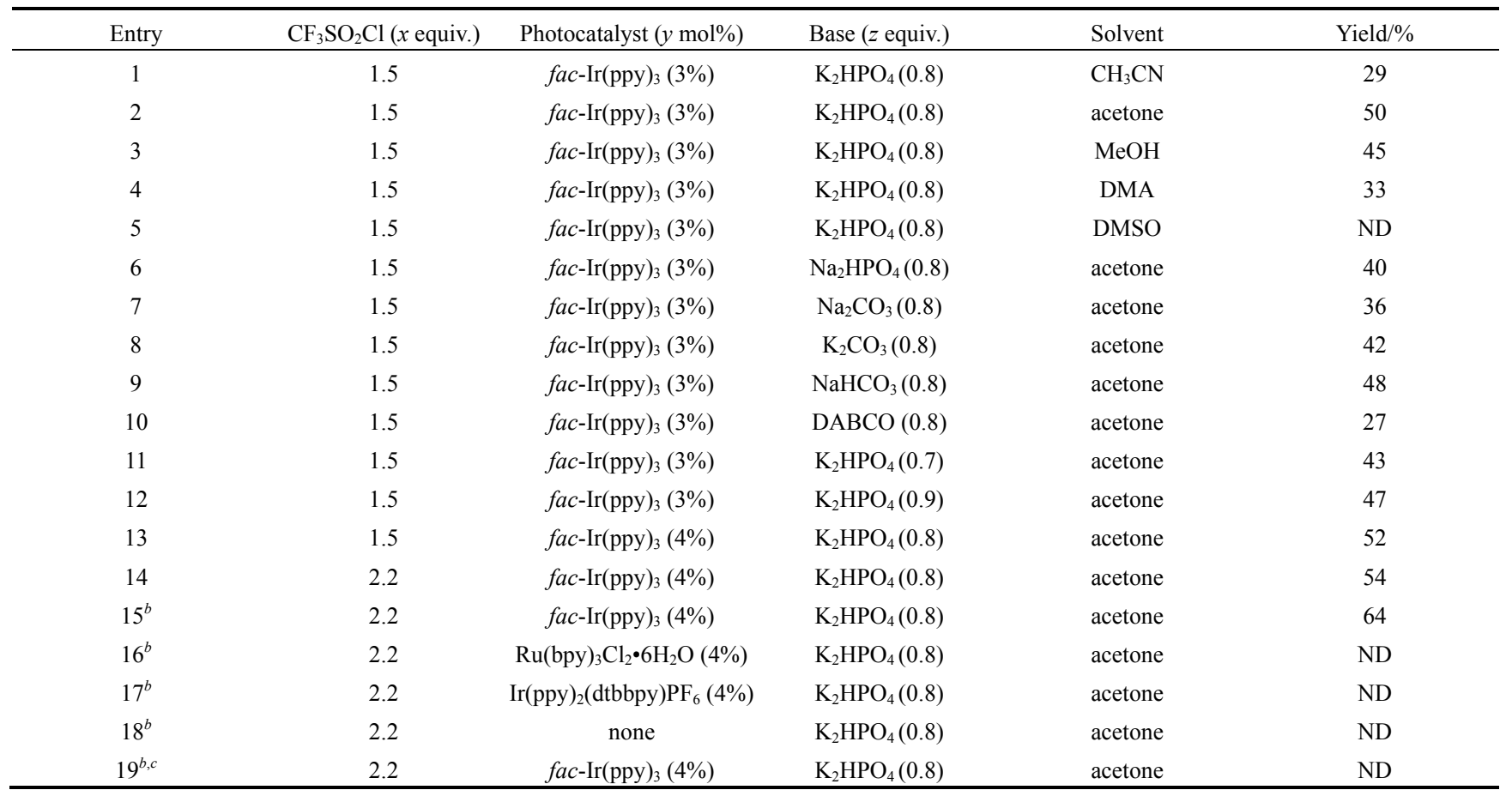

${ }^{a}$ Reaction Conditions: 1a $(0.2 \mathrm{mmol}), \mathrm{CF}_{3} \mathrm{SO}_{2} \mathrm{Cl}$, photocatalyst, additive, and solvent $(3 \mathrm{~mL})$, irradiated with $15 \mathrm{~W}$ blue LEDs at r.t. under $\mathrm{N}_{2}$ atmosphere. Yields of isolated products are given. ${ }^{b}$ The first portion of $\mathrm{CF}_{3} \mathrm{SO}_{2} \mathrm{Cl}$ (1.2 equiv.) is added and the rest (1.0 equiv.) is added after $3 \mathrm{~h} .{ }^{c}$ In dark.

\section{References}

[1] For selected reviews: (a) Chen, F.; Wang, T.; Jiao, N. Chem. Rev. 2014, 114, 8613; (b) Souillart, L.; Cramer, N. Chem. Rev. 2015, 115, 9410; (c) Song, F.; Gou, T.; Wang, B.-Q.; Shi, Z.-J. Chem. Soc. Rev. 2018, 47, 7078; (d) Wu, X.; Zhu, C. Chem. Rec. 2018, 18, 587; (e) Wu, X.; Zhu, C. Chin. J. Chem. 2019, 37, 171; (f) Wang, B.; Liu, Y.; Hao, Z.; Hou, J.; Li, J.; Li, S.; Pan, S.; Zeng, M.; Wang, Z. Chin. J. Org. Chem. 2018, 38, 1872 (in Chinese). (王柏文, 刘园, 郝志峰, 侯佳琦, 李健怡, 李舒婷, 潘思慧, 曾铭豪, 汪朝阳, 有机化学, 2018, 38, 1872); (g) Liang, T.; Jiang, L.; Gan, M.; Su, X.; Li, Z. Chin. J. Org. Chem. 2017, 37, 3096 (in Chinese). (梁婷婷, 姜岗, 干苗苗，苏金鎂，李争宁，有机化学，2017，37，3096); (h) Wu，K.; Song, C.; Cui, D. Chin. J. Org. Chem. 2017, 37, 586 (in Chinese). (吴空, 宋婵, 崔冬梅, 有机化学, 2017, 37, 586); (i) Zhong, J.-J.; Meng, Q.-Y.; Chen, B., Tung, C.-H.; Wu, L.-Z. Acta Chim. Sinica 2017, 75, 34 (in Chinese). (钟建基, 孟庆元, 陈涁, 佟振合, 吴网 珠, 化学学报, 2017, 75, 34)

[2] For selected reviews on FGM: (a) Wu, X.; Wu, S.; Zhu, C. Tetrahedron Lett. 2018, 59, 1328; (b) Li, W.; Xu, W.; Xie, J.; Yu, S.; Zhu, C. Chem. Soc. Rev. 2018, 47, 654. For selected examples from our group, see: (c) Wu, Z.; Ren, R.; Zhu, C. Angew. Chem. Int. Ed. 2016, 55, 10821; (d) Wu, Z.; Wang, D.; Liu, Y.; Huan, L.; Zhu, C. J. Am. Chem. Soc. 2017, 139, 1388; (e) Ren, R.; Wu, Z.; Huan, L.; Zhu, C. Adv. Synth. Catal. 2017, 359, 3052; (f) Ji, M.; Wu, Z.; Yu, J.; Wan, X.; Zhu, C. Adv. Synth. Catal. 2017, 359, 1959; (g) Wang, M.; Wu, Z.; Zhang, B.; Zhu, C. Org. Chem. Front. 2018, 5, 1896; (h) Yu, J.; Wang, D.; Xu, Y.; Wu, Z.; Zhu, C. Adv. Synth. Catal. 2018, 360, 744; (i) Zhang, H.; Wu, X.; Zhao, Q.; Zhu, C. Chem. Asian J. 2018, 13, 2453; (j) Chen, D.; Wu, Z.; Yao, Y.; Zhu, C. Org. Chem. Front. 2018, 5, 2370; (k) Tang, N.; Yang, S.; Wu, X.; Zhu, C.
Tetrahedron 2019, 75, 1639; (1) Chen, D.; Ji, M.; Yao, Y.; Zhu, C. Acta Chim. Sinica 2018, 76, 951 (in Chinese). (陈栋，吉梅山，姚英 明, 朱晨, 化学学报, 2018, 76, 951); (m) Ji, M.; Wu, Z.; Zhu, C. Chem. Commun. 2019, 55, 2368.

[3] For selected examples of remote FGM from other groups: (a) Thaharn, W.; Soorukram, D.; Kuhakarn, C.; Tuchinda, P.; Reutrakul, V.; Pohmakotr, M. Angew. Chem. Int. Ed. 2014, 53, 2212; (b) Li, L.; Li, Z.-L.; Wang, F.-L.; Guo, Z; Cheng, Y.-F.; Wang, N.; Dong, X.-W.; Fang, C.; Liu, J.; Hou, C.; Tan, B.; Liu, X.-Y. Nat. Commun. 2016, 7, 13852; (c) Li, Z.-L.; Li, X.-H.; Wang, N.; Yang, N.-Y.; Liu, X.-Y. Angew. Chem. Int. Ed. 2016, 55, 15100; (d) Li, L.; Li, Z.-L.; Gu, Q.-S.; Wang, N.; Liu, X.-Y. Sci. Adv. 2017, 3, e1701487; (e) Wang, N.; Li, L.; Li, Z.-L.; Yang, N.-Y.; Guo, Z.; Zhang, H.-X.; Liu, X.-Y. Org. Lett. 2016, 18, 6026; (f) Tang, X.; Studer, A. Angew. Chem. Int. Ed. 2018, 57, 814; (g) Wang, H.; Xu, Q.; Yu, S. Org. Chem. Front. 2018, 5, 2224; (h) He, Y.; Wang, Y.; Gao, J.; Zeng, L.; Li, S.; Wang, W.; Zheng, X.; Zhang, S.; Gu, L.; Li, G. Chem. Commun. 2018, 54, 7499; (i) Gu, L.; Gao, Y.; Ai, X.; Jin, C.; He, Y.; Li, G.; Yuan, M. Chem. Commun. 2017, 53, 12946; (j) Wei, X.-J.; Noël, T. J. Org. Chem. 2018, 83, 11377.

[4] Xu, Y.; Wu, Z.; Jiang, J.; Ke, Z.; Zhu, C. Angew. Chem. Int. Ed. $\mathbf{2 0 1 7}, 56,4545$.

[5] For selected examples of alkynyl migration: (a) Tang, X.; Studer, A. Chem. Sci. 2017, 8, 6888; (b) Liu, J.; Li, W.; Xie, J.; Zhu, C. Org. Chem. Front. 2018, 5, 797; (c) Zhao, Q.; Ji, X.-S.; Gao, Y.-Y.; Hao, W.-J.; Zhang, K.-Y.; Tu, S.-J.; Jiang, B. Org. Lett. 2018, 20, 3596.

[6] (a) Meadows, D. C.; Gervay-Hague, J. Med. Res. Rev. 2006, 26, 793; (b) Feng, M.; Tang, B.; Liang, S. H.; Jiang, X. Curr. Top. Med. Chem. 2016, 16, 1200; (c) Shaaban, S.; Liang, S.; Liu, N.-W.; Manolikakes, G. Org. Biomol. Chem. 2017, 15, 1947; (d) Qiu, G.; Zhou, K.; Gao, L.; Wu, J. Org. Chem. Front. 2018, 5, 691. 